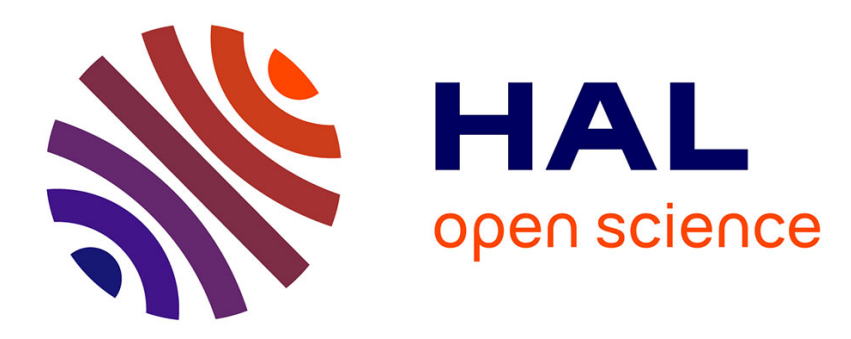

\title{
Sur la réfraction et la diffraction des rayons de Röntgen M. Gouy
}

\section{- To cite this version:}

M. Gouy. Sur la réfraction et la diffraction des rayons de Röntgen. J. Phys. Theor. Appl., 1896, 5

(1), pp.345-350. 10.1051/jphystap:018960050034501 . jpa-00239905

\section{HAL Id: jpa-00239905 https://hal.science/jpa-00239905}

Submitted on 1 Jan 1896

HAL is a multi-disciplinary open access archive for the deposit and dissemination of scientific research documents, whether they are published or not. The documents may come from teaching and research institutions in France or abroad, or from public or private research centers.
L'archive ouverte pluridisciplinaire $\mathbf{H A L}$, est destinée au dépôt et à la diffusion de documents scientifiques de niveau recherche, publiés ou non, émanant des établissements d'enseignement et de recherche français ou étrangers, des laboratoires publics ou privés. 


\section{SUR LA RÉFRAGTION ET LA DIFFRAGTION DES RAYONS DE RÖNTGEN (2);}

Par M. GOUY.

1. - Disposition d'une source linéaire. - La réfraction et la diffraction des rayons de Röntgen ont déjà fait l'objet de plusieurs recherches, qui ont donné des résultats négatifs ou douteux, mais avec une assez faible approximation. La difficulté consistait à avoir une source de radiation qui fût à la fois assez intense pour opérer photographiquement à grande distance, et d'étendue assez limitée, au moins dans un sens, pour donner des ombres bien définies. Le dispositif usité, c'est-à-dire l'emploi d'une fente et d'une source ordinaire, peut satisfaire à la seconde condition, mais c'est en réduisant l'intensité à si peu de chose, qu'il faut, avec des durées de pose acceptables, se placer à une distance trop petite pour des mesures bien exactes.

Cette difficulté, que rencontrent toutes les expériences relatives à la marche et à la propagation des radiations nouvelles, a été surmontée au moyen d'un dispositif que m'a suggéré l'étude des prcpriétés des tubes du modèle dit focus, où les rayons prennent naissance à la surface d'une lame plane de platine, placée au centre de courbure d'une cathode sphérique.

Si l'on examine, au moyen d'un écran phosphorescent ou d'ure

(1) Les abaissements moléculaires limites que j'ai obtenus ne peuvent être comparés à ceux faisant l'objet des lois de Raoult: ils n'ont pas la même signification.

Les abaissements considérés par M. Raoult sont déterminés empiriquement en prolongeant jusqu'à l'axe des ordonnées la portion de la courbe des abaissements moléculaires aux points où elle se confond sensiblement avec une droite.

Elle représente, d'après M. Raoult, l'abaissement moléculaire du corps dissous, en supposant que celui-ci conserve dans ses solutions infiniment dilućes la constitution qu'il possède aux points où la courbe a été prolongée. (Voir mon mémoire original.)

() Comptes rendus, t. CXXII, p. 1197, 26 mai ; et t. CXXIII, p. 43, 6 juillet 1896. 
plaque photographique, la répartition du rayonnement, on constate que celui-ci est distribué presque uniformément dans l'hémisphère limité par le plan de la lame de platine; ce n'est que pour les directions faisant avec ce plan un angle $\alpha$ inférieur à $2^{\circ}$ ou $3^{\circ}$ que l'on constate une diminution marquée, et, en fait, la trace de ce plan sur l'écran ou le cliché est nettement dessinée jusqu'à une assez grande distance, si la lame est bien plane ('). Cette loi, tout opposée à la loi photométrique du cosinus, montre la voie à suivre pour réaliser une source linéaire et de grande intensité.

Il suffit pour cela de se placer presque dans le plan de la lame. Si celle-ci a une portion active de diamètre $a$, la source, vue obliquement, aura pour largeur maximum $a \sin \kappa$, quantité qui peut ètre rendue très petite, surtout si l'appareil est réglé de telle sorte que $a$ soit réduit. Ainsi, pour le tube qui a servi à presque toutes mes expériences, on a sensiblement: $a=3$ millimètres ${ }^{(2)}$; et on utilise des rayons pour lesquels $\sin x=0,01$; la largeur apparente de la source ne dépasse donc pas $30 \%$.

Quant à l'intensité dans ces conditions, on peut l'évaluer à la moitié de l'intensité émise moins obliquement. L'appareil, disposé pour fonctionner indéfiniment sans avaries, donne des images vigoureuses à ŏ mètres en quatre heures, durée de pose qui a toujours été employée $\left({ }^{3}\right)$.

Le tube est placé, avec les appareils accessoires et la plaque, sur des consoles de pierre fixées à un mur, où l'on marque la trace du plan de la lame. Ce plan est rendu vertical, ainsi que les fils ou les

(1) C'est ainsi qu'on peut orienter exactement le plan de la lame, et marquer sa trace sur la table d’expérience.

$\left({ }^{2}\right) \mathbf{Y}$ compris le déplacement du point radiant, qui varie un peu selon le degré de vide.

(3) Ceci correspond à 10 minutes pour 1 mètre, ou à 6 secondes pour $0^{m}, 10$. Pour des poses de plus courte durée, on peut augmenter beaucoup l'intensité, mais ce régime forcé ne pourrait pas être maintenu plusieurs heures. La bobine de Rhumkorif' grand modèle est reliée à un transformateur Tesla, dont l'emploi est très pratique pour un travail prolongé. Le tube, réuni à une trompe, présente cette particularité que l'anode de platine est normale à l'axe du faisceau cathodique; les deux électrodes sont fixées par des bouchons (garnis de mastic et de glu marine), de manière à permettre le réglage, qui a une grande importance pour l'intensité et les dimensions de la source radiante.

La durée de quatre heures m'a paru ne pas pouvoir être dépassée utilement et pratiquement, dans les conditions ou j'opérais. Pour doubler la distance, il faudrait poser seize heures, ce qui augmente les chances de dérangements des appareils et devient gênant. 
fentes emploýées, dont le parallélisme exact avec la source linéaire est une condition nécessaire.

2. - Expériences sur la réfraction. - Deux fils de platine de $40 \mu$ de diamètre, recuits et fortement tendus, sont placés parallèlement l'un à l'autre, à une distance de 2 millimètres. Deux prismes de même angle et de même substance sont disposés contre les fils, vers leur milieu; le tout est à égale distance de la source et de la plaque photographique.

L'ombre des fils se montre sur le cliché sous l'apparence de deux lignes claires, assez bien définies pour supporter un certain grossissement, et d'une largeur de 100 ${ }^{\mu}$ environ. Les deux extrémités A et B de cet ensemble sont formées par les rayons qui n'ont traversé que l'air; le milieu C est formé parles rayons qui ont traverséles prismes, et présente, en général, une intensité moindre.

L'expérience consiste à examiner si ces lignes sont droites; pour cela on mesure la distance $\delta$ des deux lignes, en divers points des portions $\mathrm{A}, \mathrm{B}$ et $\mathrm{C}$, symétriquement placés par rapport à un point pris vers le milieu de la région C. Les prismes sont disposés en sens inverse, de manière que leurs arêtes réfringentes soient entre les fils. En appelant $\delta_{1}$ la moyenne des mesures faites dans les régions $\mathrm{A}$ et $\mathrm{B}$, et $\delta_{2}$ la moyenne des mesures faites dans la région $\mathrm{C}$, l'écart $\varepsilon$ produit par un seul prisme sera :

$$
\varepsilon=\frac{1}{2}\left(\hat{o}_{2}-\widehat{o}_{1}\right) \text {. }
$$

et sera du signe de $n-1$, en appelant $n$ l'indice des prismes par rapport à l'air. On élimine ainsi, comme on le voit, l'effet d'un défaut de parallélisme des fils. Les mesures sont faites au moyen d'un appareil construit par MM. Brünner pour l'étude micrométrique des clichés ( ${ }^{1}$ ), consistant en deux chariots rectangulaires, munis de. règles divisées et de microscopes micrométriques. Un des chariots porte le cliché et ne sert ici qu'à définir les points où se font les mesures; l'autre sert à mesurer $\delta$. Les pointés sur le cliché se font avec un grossissement de dix fois, et comportent une erreur de quelques microns; les pointés sur la règle se font avec une très

(1) Cet appareil, construit à l'occasion du passage de Vénus, est figuré dans un mémoire de I. Vautier (Annales de Chimie et de Physique, $6^{\text {e }}$ série, t. Xv, p. 327). Je dois noter ici qu'on peut éliminer l'effet fâcheıx du grain du gélatino-bromure en faisant usage d'une lentille cylindrique placée sur loculaire. 
grande exactitude. On a soin, dans le cas actuel, de faire toutes les mesures en utilisant les mêmes traits de la règle et les mêmes portions de la vis du microscope, ce qui est aisé, les deux lignes à étudier étant droites et parallèles à quelques microns près.

Avant toute expérience, il faut s'assurer que le défaut de rectitude des fils n'introduira pas d'erreur sensible. Pour cela, au moyen du même appareil muni d'un fort grossissement, on mesure la distance des fils, d'axe en axe. J'ai constaté que l'erreur de ce chef sur $\varepsilon$ ne peut dépasser $3^{\mu}$; cette erreur, du reste, n'ayant pas un caractère systématique, doit disparaître en majeure partie dans les moyennes de mesures faites en des points différents, et il m'a paru inutile d'essayer d'en tenir compte.

Voici le tableau des valeurs de $\varepsilon$ mesurées sur les divers clichés. Deux séries ont été faites (1) : la première, avec des corps assez transparents, comporte des prismes de $60^{\circ}$ (une face normale au rayon) et une distance de 5 mètres entre la source et le cliché; dans la seconde, portant sur des substances presque opaques, l'angle a été réduit à $30^{\circ}$, et la distance à $0^{\mathrm{m}},{ }^{\circ} 0\left(^{(2)}\right.$.

\begin{tabular}{|c|c|c|c|c|}
\hline \multicolumn{2}{|c|}{ No du eliché } & Substance & $\varepsilon$ (microns) & $(n-1) \cdot 10^{\circ}$ \\
\hline \multirow{7}{*}{$1^{\text {re }}$ série } & 1 & Aluminium & -2 & $-0,46$ \\
\hline & 2 & Crown-glass & $-\tilde{3}$ & $-0,69$ \\
\hline & 3 & Ébonite & -4 & $-0,92$ \\
\hline & 4 & $I d$. & $-0,5$ & $+0,12$ \\
\hline & $\ddot{z}$ & Soufre & +3 & $+0,69$ \\
\hline & 6 & Cire viergẹ & +3 & $+0,69$ \\
\hline & 7 & $I d$. & -2 & $-0,09$ \\
\hline \multirow{3}{*}{$2^{\mathrm{c}}$ série } & 8 & Flint-glass & +2 & $+7,0$ \\
\hline & 9 & Fer & -5 & $-17,3$ \\
\hline & 10 & Zine & +3 & $+10,5$ \\
\hline
\end{tabular}

Il résulte de ce tableau que, pour les corps transparents, les dévia. tions observées ne sont que des fractions de seconde $\left(1^{\prime \prime}=12^{\mu}\right)$, et

(1) Je ne parle pas jci d'expériences préliminaires, qui conduisaient aux mêmes conclusions, arec une précision moindre.

${ }^{(2)}$ Exceptionnellement, le cliché $n^{\circ} 7$ a été fait avec dix prismes, cinq sur chaque fil, et le cliché $n^{\circ} 4$ a été fait avec une disposition des prismes anormale, les arêtes réfringentes étant en dehors des deux fils, en sorte que $\varepsilon$ soit de signe opposé à $n-1$.

Dans la deuxième série, des lames de métal ont été placées sur les portions non courertes pas les prismes, qui, sans cela, auraient été surexposées. 
que l'indice dépasse l'unité de moins de $\frac{1}{1000000}$. Les écarts sont trop petits pour qu'on puisse en répondre (') ; nous conclurons donc que la réfraction est insensible actuellement, et qu'il faudrait la rechercher dans la septième décimale de l'indice.

Pour la deuxième série, les écarts ne dépassent pas non plus les erreurs admissibles, mais l'approximation $\operatorname{sur} n-1$ est environ vingt fois moindre.

3. - Expériences sur la diffraction. - Dans les conditions des expériences précédentes, la lumière ne donnerait qu'une ombre extrêmement faible, et large de plusieurs centimètres. Cette remarque nous montre que la diffraction est nulle ou peu marquée pour les rayons de Röntgen, comme on le savait déjà. Je me suis proposé de soumettre la question à une épreuve rigoureuse, et jai fait choix du phénomène de l'épanouissement du faisceau passant par une fente étroite, qui présente cet avantage que toutes les erreurs d'expérience tendent à augmenter encore l'élargissement.

Je rapporterai, entre autres, l'expérience suivante. Une fente de $43^{\mu}$ de largeur $\left(^{2}\right)$ est placée à $2^{\mathrm{m}}$, ö0 de la plaque, qui est elle-même à 5 mètres de la source linéaire. Si aucune complication n'intervenait, on aurait une image nette de $90^{\mu}$ de largeur.

En réalité, les dimensions de la source, le grain du cliché et les petits déplacements des appareils pendant la pose de quatre heures empêchent que l'image ait des bords absolument définis, mais l'examen micrométrique montre que cette image presque tout entière est comprise dans une largeur de $110^{\mu}$ à $120^{\mu}$, ce qui est en dehors étant peu de chose, et assurément moins de $\mathbf{1} / \mathbf{4}$ de l'intensité maximum.

Supposons maintenant que la diffraction existe comme pour la lumière, et que la longueur d'onde soit $0 \mu, 00$. En calculant les intensités à des distances $x$ du milieu de l'image, et admettant que la source ait une largeur nulle, on a les résultats suivants :

(1) Ce genre d'expériences comporte une erreur systématique, si l'absorption des prismes est assez marquée pour que le champ soit d'inégale intensité aux deux bords de l'ombre du fil. Ici, en raison du peu de largeur de cette ombre, cette inégalité était absolument inappréciable, du moins dans la première série.

() La fente, en laiton, de 2 millimètres d'épaisseur, présente des bords soigneusement travaillés et épais (non en biseau), ce qui est nécessaire, en raison de limparfaite opacité dı métal. L'orientation exacte de la fente se fait aisément en remplaçant le tube par une source lumineuse.

J. de phys., $3^{\circ}$ série, t. V. (Août 1896.) 
350

PERRIN

$\begin{array}{cc}x \text { (microns) } & \text { Intensité } \\ 0 & 1 \\ 45 & 0,91 \\ 90 & 0,70 \\ 135 & 0,44 \\ 180 & 0,21 \\ 225 & 0,06\end{array}$

Ces nombres définissent une bande lumineuse dont la largeur serait estimée assurément très supérieure à la précédente ; ainsi, en s'arrêtant aux points où l'intensité est $1 / 4 \mathrm{du}$ maximum, on trouverait une largeur de $340^{\mu}$, soit 3 fois celle qui a été mesurée.

Il est donc établi d'une manière certaine que, si la diffraction existe comme pour la lumière, et qu'on puisse parler de la longueur d'onde des nouveaux rayons, celle-ci est considérablement inférieure à $0^{\mu}, 005$, qui vaut $\frac{1}{100}$ de la longueur d'onde du vert.

Rien, du reste, n'indique qu'il existe une diffraction quelconque, le petit excès de largeur de l'image sur la largeur théorique $\left(90^{\mu}\right)$, s'expliquant naturellement par les causes rappelées plus haut. 